\title{
Article \\ High-Level Carbapenem Resistance among OXA-48-Producing Klebsiella pneumoniae with Functional OmpK36 Alterations: Maintenance of Ceftazidime/Avibactam Susceptibility
}

\author{
Pilar Lumbreras-Iglesias ${ }^{1,2} \mathbb{D}^{\infty}$, María Rosario Rodicio ${ }^{2,3}{ }^{\mathbb{D}}$, Pablo Valledor ${ }^{4}$, Tomás Suárez-Zarracina ${ }^{5}$ \\ and Javier Fernández $1,2,4, *$ (D) \\ 1 Department of Clinical Microbiology, Hospital Universitario Central de Asturias, 33011 Oviedo, Spain; \\ pilar.lumbreras08@gmail.com \\ 2 Traslational Microbiology Group, Instituto de Investigación Sanitaria del Principado de Asturias (ISPA), \\ 33011 Oviedo, Spain; rrodicio@uniovi.es \\ 3 Department of Functional Biology, Microbiology Area, University of Oviedo, 33006 Oviedo, Spain \\ 4 Research \& Innovation, Artificial Intelligence and Statistical Department, Pragmatech AI Solutions, \\ 33003 Oviedo, Spain; pablo.valledor@pragmatech.ai \\ 5 Department of Internal Medicine, Hospital Universitario Central de Asturias, 33011 Oviedo, Spain; \\ tomassecades@hotmail.com \\ * Correspondence: javifdom@gmail.com
}

Citation: Lumbreras-Iglesias, P.; Rodicio, M.R.; Valledor, P.; Suárez-Zarracina, T.; Fernández, J. High-Level Carbapenem Resistance among OXA-48-Producing Klebsiella pneumoniae with Functional OmpK36 Alterations: Maintenance of Ceftazidime/Avibactam Susceptibility. Antibiotics 2021, 10, 1174. https://doi.org/10.3390/ antibiotics10101174

Academic Editor: Francesca Andreoni

Received: 17 August 2021

Accepted: 22 September 2021

Published: 27 September 2021

Publisher's Note: MDPI stays neutral with regard to jurisdictional claims in published maps and institutional affiliations.

Copyright: (c) 2021 by the authors. Licensee MDPI, Basel, Switzerland. This article is an open access article distributed under the terms and conditions of the Creative Commons Attribution (CC BY) license (https:/ / creativecommons.org/licenses/by/ $4.0 /)$.

\begin{abstract}
The aim of this work was to analyze outer membrane porin-encoding genes (ompK35 and ompK36) in a collection of OXA-48 producing Klebsiella pneumoniae, to assess the effect of porin alterations on the susceptibility to ceftazidime/avibactam, and to describe a screening methodology for phenotypic detection of OXA-48-producing K. pneumoniae with disrupted porins. Antimicrobial susceptibility was tested by Microscan and Etest. The genomes of 81 OXA-48-producing K. pneumoniae were sequenced. MLST, detection of antimicrobial resistance genes, and analysis of ompK35 and ompK36 were performed in silico. Tridimensional structures of the OmpK36 variants were assessed. Receiver operating characteristics curves were built to visualize the performance ability of a disk diffusion assay using carbapenems and cefoxitin to detect OmpK36 functional alterations. A wide variety of OmpK36 alterations were detected in 17 OXA-48-producing K. pneumoniae isolates. All displayed a high-level meropenem resistance (MIC $\geq 8 \mathrm{mg} / \mathrm{L}$ ), and some belonged to high-risk clones, such as ST15 and ST147. Alterations in ompK35 were also observed, but they did not correlate with high-level meropenem resistance. All isolates were susceptible to ceftazidime/avibactam and porin alterations did not affect the MICs of the latter combination. Cefoxitin together with ertapenem/meropenem low inhibition zone diameters (equal or lower than $16 \mathrm{~mm}$ ) could strongly suggest alterations affecting OmpK36 in OXA-48-producing K. pneumoniae. OXA-48-producing K. pneumoniae with porin disruptions are a cause of concern; ceftazidime/avibactam showed good in vitro activity against them, so this combination could be positioned as the choice therapy to combat the infections caused by this difficult-to-treat isolates.
\end{abstract}

Keywords: K. pneumoniae; carbapenem resistance; OXA-48; porins; ceftazidime/avibactam

\section{Introduction}

The use of carbapenems has been increasing in hospitals in the past years as a consequence of the rise of extended spectrum $\beta$-lactamase (ESBL) and/or AmpC-producing Enterobacterales [1]. The extensive clinical use of these drugs has led in turn to the emergence of carbapenem-resistant Enterobacterales (CRE), boosted by the spread of carbapenemases, such as serine $\beta$-lactamases (KPC and OXA-48) or metallo- $\beta$-lactamases (VIM, IMP and NDM), by means of mobile genetic elements [2]. Carbapenem resistance can also be mediated by permeability defects in the outer membrane of ESBL- or AmpC-producing strains [3], which can besides increase the minimal inhibitory concentrations (MICs) of 
carbapenems in carbapenemase-producing Enterobacterales (CPE). These defects can be associated with alterations in porin-encoding genes or their promoter regions, such as point mutations, deletions or insertions which can hinder the synthesis of a functional protein, hence obstructing the entry of molecules into the periplasm [4]. Klebsiella pneumoniae is one of the main reservoirs of carbapenemases in the hospital environment [5]. OmpK35 and OmpK36 are the major porins in this species, and they constitute the main pathway through which carbapenems enter the cell [6].

The increasing carbapenem resistance among Enterobacterales poses a major threat to public health because of its difficult treatment. Several clinical studies have demonstrated that carbapenems, alone or combined with other drugs, are options for the treatment of infections caused by CRE when MICs of these compounds are low [7,8]. This is the case of most infections caused by OXA-48-producing Enterobacterales, which weakly hydrolyze carbapenems $[9,10]$. However, when MICs of meropenem are higher than $8 \mathrm{mg} / \mathrm{L}$, the probability of reaching therapeutic success is low according to stochastic modeling data, and thus carbapenems are contraindicated [7]. In response to the emergence and spread of CRE, new antimicrobials or combinations of a $\beta$-lactam plus a $\beta$-lactamase inhibitor are either under development or have been approved in the last years. This is the case of ceftazidime/avibactam, a combination of a third-generation cephalosporin with a synthetic $\beta$-lactamase inhibitor that prevents the activities of Ambler class A and $C \beta$-lactamases and some Ambler class D enzymes including OXA-48 [11].

In view of the worrisome medical situation raised by $C R E$, and the recognized contribution of porin alterations to carbapenem resistance, the aims of the present study were (i) to molecularly analyze outer membrane porin-encoding genes in a collection of OXA-48 producing K. pneumoniae isolates; (ii) to assess the effect of the observed alterations on the susceptibility to ceftazidime/avibactam; (iii) to develop a screening methodology for phenotypic detection of OXA-48 producing Klebsiella spp. with porin disruptions.

\section{Results and Discussion}

\subsection{Carbapenems and Ceftazidime/Avibactam Susceptibility, $\beta$-Lactamases and Molecular Epidemiology}

Bacterial identification by MALDI-TOF recognized the 81 isolates as K. pneumoniae, results which were confirmed in silico by analysis of the sequenced genomes. Strains of K. pneumoniae were classified in 15 sequence types (ST), being ST326 (22/81, 27.2\%) and ST147 (20/81, 24.7\%) the most prevalent (Table 1 and Table S1). The presence of bla OXA-48 was confirmed in all of them. Antimicrobial susceptibility testing, performed with the Microscan system, showed that $100 \%, 24.69 \%$ and $20.99 \%$ isolates were resistant to ertapenem, imipenem and meropenem, respectively. In addition to the five isolates selected from other samples, twelve recovered from blood cultures displayed high level of meropenem resistance (MIC $\geq 8 \mathrm{mg} / \mathrm{L}$ ), which could not be explained only by the OXA-48 production. This suggested the existence of additional mechanisms. Apart from $\beta$-lactamase production, mechanisms which can lead to a high-level carbapenem resistance comprise efflux pumps, PBP (penicillin binding protein) alterations, and mutations that modify the expression and/or function of outer membrane porins, the latter being a mechanism frequently found in Enterobacterales [6]. All isolates of the study were susceptible to ceftazidime/avibactam, including those highly resistant to meropenem, with MICs ranging from $0.19 \mathrm{mg} / \mathrm{L}$ to $0.75 \mathrm{mg} / \mathrm{L}$ (three of the latter did not coproduced ESBLs and were also susceptible to ceftazidime alone). Relevant features of the 17 isolates displaying high-level carbapenem resistance are shown in Table 1, while the same features relative to the remaining isolates are compiled in Supplementary Table S2. 
Table 1. Microbiological features of OXA-48-producing Klebsiella pneumoniae isolates displaying high carbapenem resistance due to porin alterations.

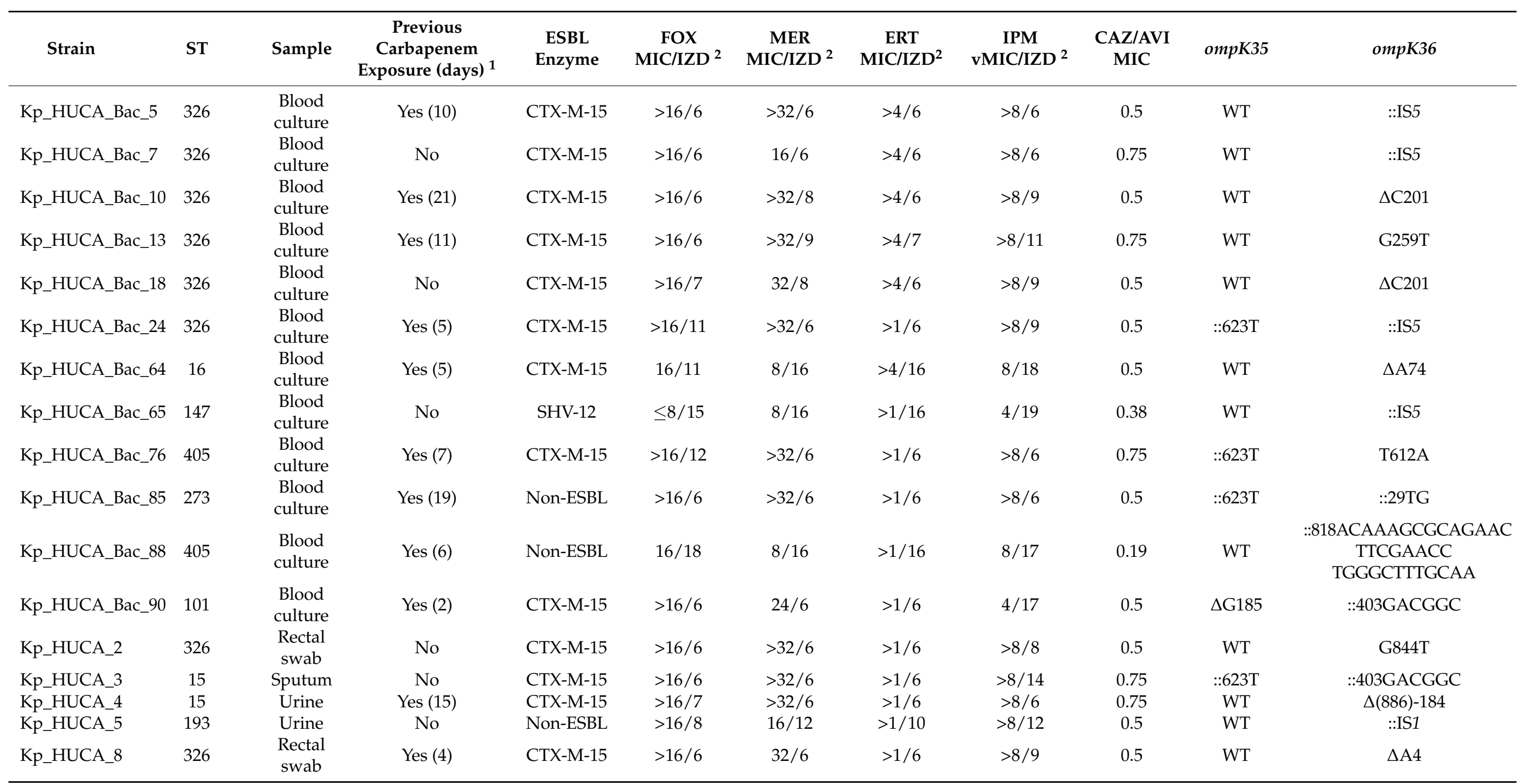

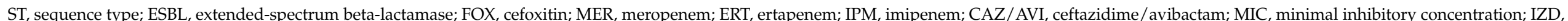

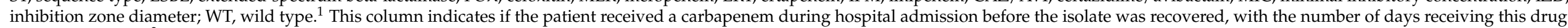
shown in brackets. ${ }^{2}$ MIC are expressed in $\mathrm{mg} / \mathrm{L}$ and IZD in $\mathrm{mm}$. 
Moreover, $82 \%$ of the isolates displaying high-level carbapenem resistance (14/17) coproduced ESBLs, with roughly the same rate (52/64, 81\%) found among the isolates with low carbapenem MICs (MICs $\leq 4 \mathrm{mg} / \mathrm{L}$ ). All of the isolates which did not coproduce ESBLs were susceptible to ceftazidime alone, including those three that displayed high-level carbapenem resistance. $b l a_{\mathrm{SHV}-12}$ and $b l a_{\mathrm{CTX}-\mathrm{M}-15}$ were the most frequent genes detected among the isolates that showed an ESBL phenotype (Table 1 and Table S1). Based on the antimicrobial susceptibility results, and considering that three of the highly-carbapenem resistant isolates did not co-produce ESBLs, there does not appear to be a necessary correlation between production of these enzymes and high MICs of meropenem. For this reason, a detailed analysis of the genes encoding the main porins of K. pneumoniae, OmpK35 and OmpK36, and their upstream regions was performed, in order to infer possible permeability alterations in the outer cell membrane.

\subsection{Molecular Analysis of OmpK35 and OmpK36 in OXA-48 Producers}

Bioinformatic analysis of ompK35 from the 17 isolates with high-level carbapenem resistance revealed a single nucleotide insertion ( $\mathrm{T}$ ) at 623 position in four of them, and a single nucleotide deletion $(\mathrm{G})$ at 185 position in another one (Table 1). Both alterations led to a change in the reading frame. On the other hand, analysis of the ompK35 sequences of the isolates exhibiting low meropenem MICs showed that three of them carried a IS1-like element inserted upstream of the gene, one had a G890A transition in the coding region, which resulted in a premature stop codon, and another one had a single nucleotide deletion (G) at position 575 (Table S1). Accordingly, it seems that at least not all the changes detected in ompK35 are enough to substantially increase carbapenem MICs (Table S1). These results are in line with previous observations by other authors, reporting that alterations affecting ompK35 are not capable to generate high resistance to carbapenems [12-14].

Regarding OmpK36, functional alterations have been broadly reported among KPCproducing K. pneumoniae [15-17]; however, reports and analysis of porin disruptions among OXA-48 producers are still scarce [18]. In the present study, all isolates displaying high meropenem MICs, including the 12 strains recovered from blood cultures and the five strains selected from other samples, had alterations affecting the OmpK36 coding region or the upstream DNA, which could lead to non-functional proteins or hinder the expression of the gene (Table 1 and Figure 1). The remaining isolates carried no mutations or had nucleotide changes considered as polymorphisms.

Among high-level carbapenem resistant isolates, four contained an IS5-like element located within the coding region (three isolates) or immediately upstream of the RSB (ribosome binding site; one isolate) of OmpK36; the latter alteration was similar to one previously reported in KPC-2-producing K. pneumoniae ST258, where it was shown to be associated with significantly lower OmpK36 expression levels and increased MICs of carbapenems [15]. A single isolate carried an IS1-like insertion within OmpK36. Additional alterations included: (i) nonsense mutations that generate premature stop codons yielding truncated and probably non-functional proteins (3 isolates); (ii) single nucleotide deletions that led to frameshift mutations (4 isolates, with two of them, both ST326, having the same deletion at the same position); (iii) a large deletion of 886 nucleotides including the first 702 nucleotides of the ORF and 184 of the upstream DNA (1 isolate; not shown in Figure 1); (iv) insertions of 2 and 34 nucleotides at positions 29 and 818, respectively, which resulted on a frame shift (each displayed by a single isolate); (v) insertion of 6 nucleotides at position 403, resulting in the incorporation of two additional amino acids (DG) on a highly conserved region (L3) of the porin (two isolates). The same insertion was associated with carbapenem resistance in KPC-2-producing K. pneumoniae ST423 and ST11 isolates [16]. In addition, Lunha et al. reported a very similar amino acid insertion (GD) at the same position within the L3 region of OmpK36 in ST37 and ST11 OXA-48-producing K. pneumoniae, and linked this alteration to an increase in the carbapenem resistance level. Such resistance increase was also observed in KPC-2-producing K. pneumoniae ST258 strains with the same 
GD insertion in OmpK36, and attributed to a diminishment in the pore diameter caused by the two additional amino acids [17].

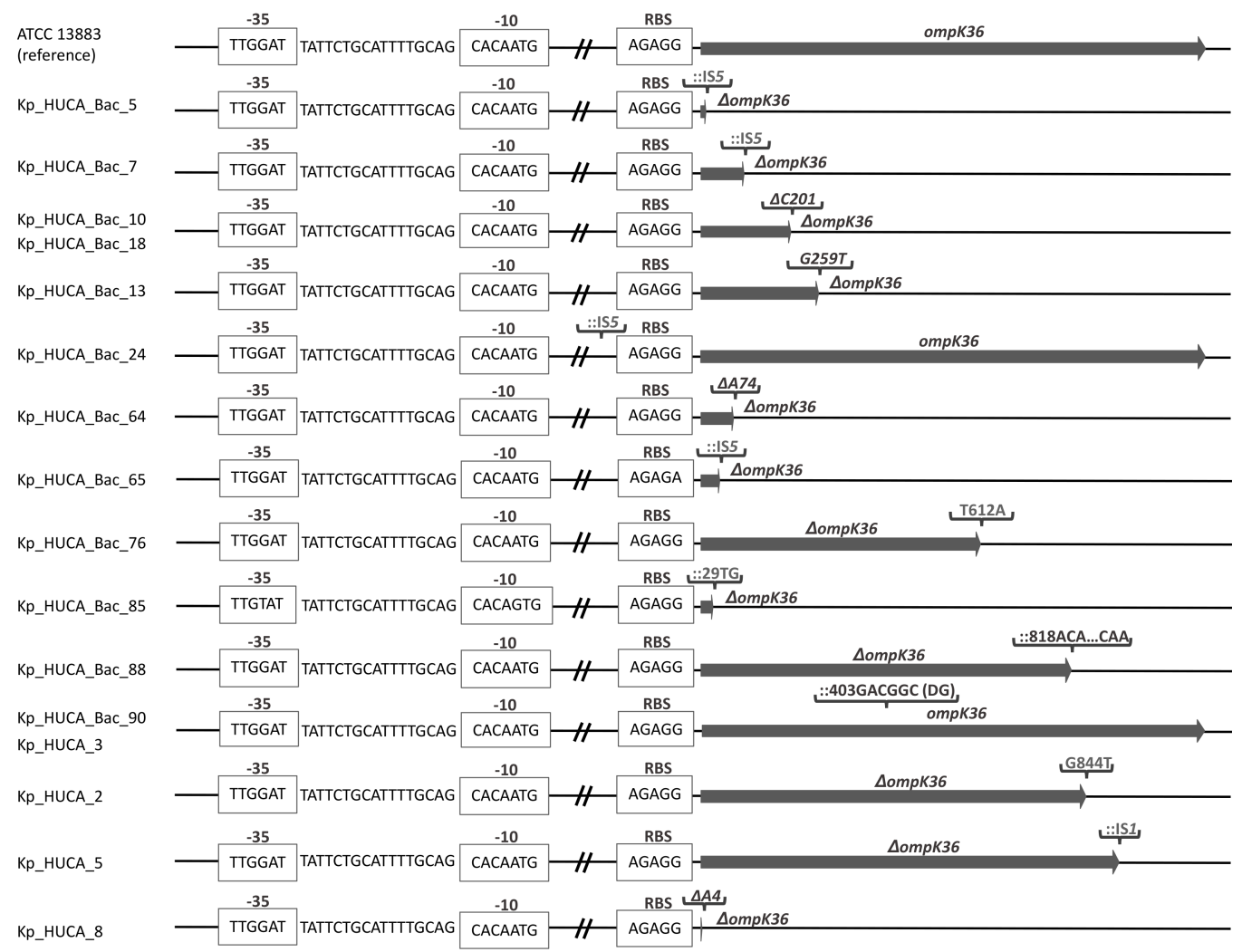

Figure 1. Genetic alterations in ompK36 and upstream DNA found in OXA-48-producing Klebsiella pneumoniae isolates displaying high-level resistance to meropenem. Kp_HUCA_4 is not represented as it carries a large deletion that includes the promoter region and the first 702 nucleotides of the ORF. RBS, ribosome binding site; IS, insertion sequence; insertion; $\Delta$, deletion.

The tridimensional structures of OmpK36 variant proteins predicted by the Phyre2 web portal are shown in Figure S1. In most of them, it can be visually verified how the conformation of the porin is affected by the observed alterations.

Our series represent a wide variety of OmpK36 alterations detected among different clones of OXA-48-producing K. pneumoniae. Some of them, such as ST15 or ST147, are considered of high-risk since they are clones which have a special ability for successful expansion, and are typically involved in outbreaks $[19,20]$, which poses an additional concern. OmpK36 disruptions described in our work are probably responsible for the high MICs of meropenem of the strains in which they were detected, in comparison with MICs of strains with functional OmpK36 proteins. The increase of meropenem MICs in OXA-48 producers is especially marked since, as previously indicated, this enzyme by itself only causes weak carbapenem hydrolysis and a consequent low level of resistance to these drugs $[9,10]$. Carbapenems must first penetrate the outer membrane in order to reach the PBPs and, because these drugs are relatively hydrophilic, their entry occurs through the water-filled porin channels [21]. Obviously, their inactivation via periplasmic carbapenemases will be more effective in increasing resistance if the influx is decreased through the loss of porins [22,23]. Apart from allowing carbapenems to cross the outer membrane, OmpK36 constitutes the entry way into the cell of some nutrients and other physiological important substances; thus, the loss of this porin is not free of charge. In fact, Wong et al. recently demonstrated that OmpK36-mediated carbapenem resistance attenuates the virulence of K. pneumoniae ST258 [17]. 
Selection of isolates displaying high-level carbapenem resistance due to outer membrane alterations may be conditioned by several factors, including the selective pressure exerted by previous exposure to carbapenems of individual patients or by the hospital environment [17]. In this sense, $11(64.7 \%)$ of the isolates with high-level carbapenem resistance in our study were recovered from patients who had been previously treated with carbapenems during the same hospital stay (Table 1). The high-level of meropenem resistance displayed by these isolates (meropenem MICs $\geq 8 \mathrm{mg} / \mathrm{L}$ ), exclude these drugs as therapeutic alternatives to treat the infections they cause. In fact, as already mentioned in the introduction, PK/PD modeling has shown that the probability of reaching the target pharmacodynamic parameter is low, even if high dose and extended infusion are administered [7]. Fortunately, all isolates in the present study were susceptible to ceftazidime/avibactam and displayed low MICs of these drugs, regardless of the OmpK36 variant. This might be due to the fact that, unlike other $\beta$-lactamase inhibitors, the outer membrane porins are not the major route by which avibactam enters the periplasm [24], and it has been previously reported that ceftazidime MICs are not affected by OmpK36 alterations [14]. Wong et al., also, did not find a decrease in the susceptibility to ceftazidime/avibactam among K. pneumoniae ST258 with alterations affecting OmpK35 or OmpK36 [17].

\subsection{Disk Diffusion Assay Using Carbapenems and Cefoxitin for Detection of Functional Alteration in OmpK36:}

Detection of strains with functional alterations in porins among OXA-48-producing $K$. pneumoniae may be of particular interest in clinical microbiology laboratories, especially when affecting OmpK36. However, next-generation sequencing techniques are not available in the routine of these laboratories, so the establishment of a phenotypic screening for the detection of such strains would be very useful to predict genotypes from phenotypes and, also, for epidemiological purposes. Most of the isolates with alterations in OmpK36 displayed reduced inhibition zone diameters to cefoxitin and carbapenems. Thus, our data suggest that resistance to both drugs could be considered a surrogate marker of functional alterations in OmpK36 in OXA-48-producing K. pneumoniae. In addition to carbapenems, it is well known that this porin constitutes the main pathway through which cefoxitin penetrates the outer membrane [4], and alterations affecting OmpK36 have been associated with the development of resistance to this drug [25]. In order to set a threshold to detect isolates carrying functional alterations in OmpK36, a disk diffusion assay testing ertapenem, imipenem, meropenem and cefoxitin was performed, and ROC curves were built to evaluate sensitivity and specificity of their detection at different thresholds (Figure 2). All isolates displaying inhibition zone diameter lower or equal to $16 \mathrm{~mm}$ to both ertapenem and meropenem, carried alterations in OmpK36 with or without changes in OmpK35. However, isolates displaying higher diameters did not have alterations in the former porin. Accordingly, the sensitivity and specificity for detection of OmpK36 functional alterations was of $100 \%$ when applying this threshold. In contrast, when using an imipenem and cefoxitin disk diffusion assay, the strains with alterations in OmpK36 could not be clearly separated from those with the wild type porin (i.e., 100\% sensitivity and specificity were not obtained at any threshold). Nevertheless, as mentioned above, a reduction on the inhibition zone to cefoxitin, together with an inhibition zone diameter to meropenem and ertapenem lower or equal to 16 , strongly suggest alterations in this porin. 
a)
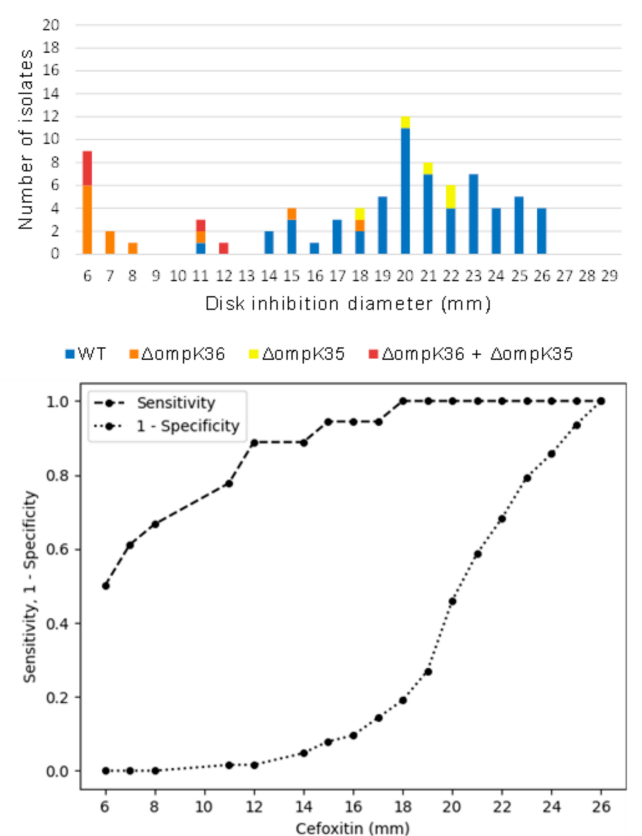

c)

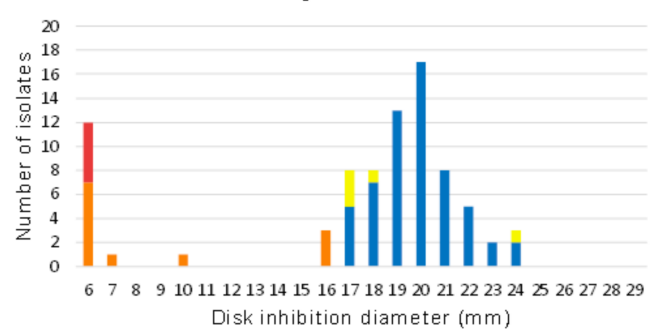

$=W T=\Delta 0 m p K 36 \quad=\Delta 0 m p K 35 \quad=\Delta 0 m p K 36+\Delta o m p K 35$

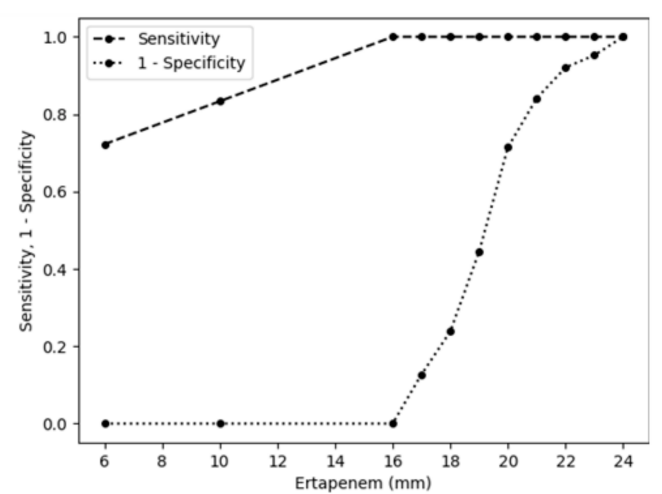

b) Meropenem
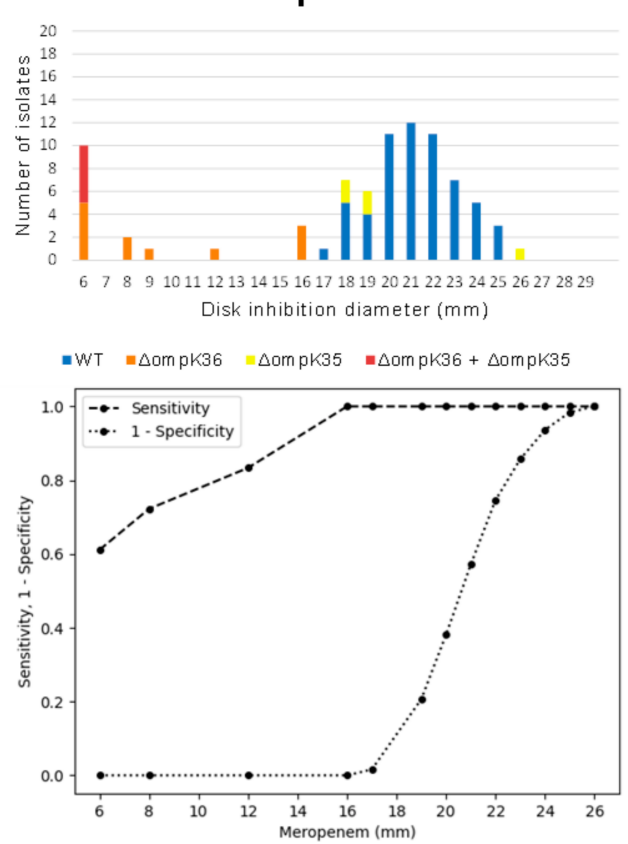

d)

Imipenem

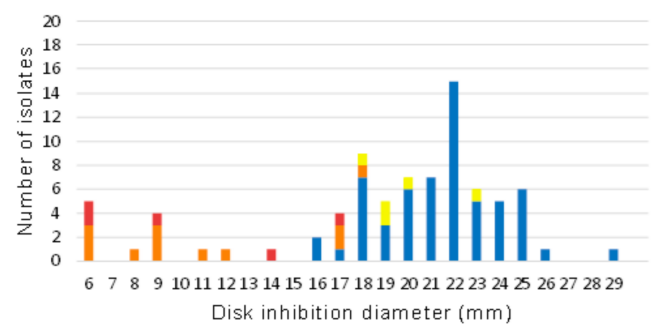

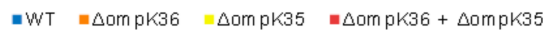

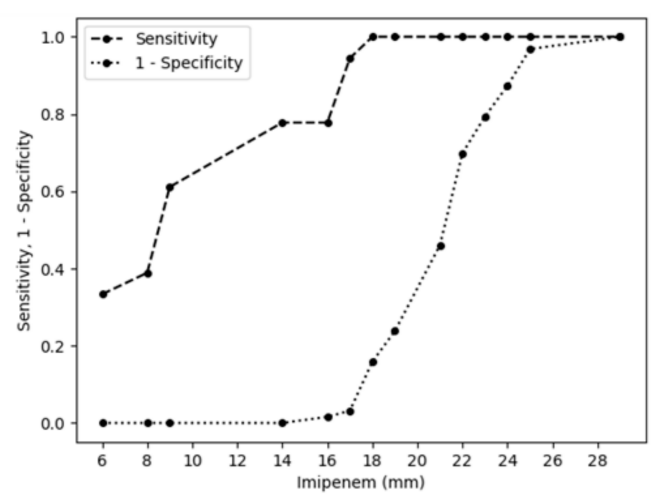

Figure 2. Distribution of cefoxitin $30 \mu \mathrm{g}(\mathbf{a})$, meropenem $10 \mu \mathrm{g}(\mathbf{b})$, ertapenem $10 \mu \mathrm{g}$ (c) and imipenem $10 \mu \mathrm{g}(\mathbf{d})$ inhibition zones (mm) of OXA-48 producing Klebsiella pneumoniae with and without porin alterations. Below of each graphic, a receiver operating curve (ROC), showing the evolution of sensitivity and 1-specificity to identify OmpK36 functional alterations in relation to the different thresholds applied for each antibiotic, is represented.

\section{Materials and Methods}

All carbapenem-resistant K. pneumoniae isolates recovered from blood cultures of different patients admitted to a tertiary hospital, Hospital Universitario Central de Asturias (HUCA), in northern Spain over a five-year period (2014-2019) were collected ( $n=76$ ). Additionally, five K. pneumoniae isolates with high meropenem MICs, recovered from different 
samples of patients admitted to the HUCA during the same period were studied. Bacterial identification was performed by MALDI-TOF/MS (Bruker Daltonics, Billerica, MA) using $\alpha$-Cyano-4-hydroxycinnamic acid as a matrix and following the manufacturer instructions available on https:/ / www.bruker.com/en/services/training, (accessed on 14 December 2020). In silico identification was performed by using KmerFinder, available at the Center for Genomic Epidemiology site (https:/ / www.genomicepidemiology.org/; CGE, 2020; accessed on 25 January 2021). Antimicrobial susceptibility was determined by the Microscan system (Beckman Coulter, Brea, CA, USA) and, also, by Etest ${ }^{\circledR}$ (bioMérieux, Marcy l'Etoile, France) for meropenem and ceftazidime/avibactam. Results were interpreted according to the EUCAST guidelines [26]. Screening of carbapenemases was performed by means of a previously described algorithm [27].

Genomic DNA from the 81 isolates (76 recovered from blood cultures and five from other samples) was extracted with the NZY Microbial gDNA Isolation kit (NZYTech, Lisbon, Portugal), and then sequenced by Illumina technology to generate 125 bp pairedend reads in a HiSeq 1500. Quality control of the reads was performed using FastQC software (Babraham Bioinformatics, Cambridgeshire, UK) and de novo assembly was carried out with VelvetOptimizer [28].

Multi-locus sequence types and the presence of resistance genes were determined in silico by the use of the MLST 2.0 and ResFinder 3.2 tools, respectively [29]. The sequences of the two major porin-encoding genes of Klebsiella (ompK35 and ompK36), including their upstream regions, were analyzed by bioinformatic tools, such as Clone Manager Professional v9.2 (CloneSuit9), Clustal Omega and Jalview [30,31]. PCR amplification of ompK36 followed by Sanger sequencing was performed using previously described primers [15], when required for confirmation of whole genome sequencing results. The tridimensional structures of OmpK36 variants were assessed on the Phyre2 web portal for protein modelling, prediction and analysis, except for sequences of less than 30 amino acid residues, which are excluded by Phyre2 specifications.

Receiver operating characteristic (ROC) curves were built to visualize the performance ability of a disk diffusion assay using ertapenem $(10 \mu \mathrm{g})$, meropenem $(10 \mu \mathrm{g})$, imipenem $(10 \mu \mathrm{g})$ and cefoxitin $(30 \mu \mathrm{g})$ (Bio-Rad, Hercules, CA, USA), to detect OmpK36 functional alterations. In order to identify the optimal cut-off values to discriminate strains carrying functional alterations in this porin, a figure showing the evolution of sensitivity and 1specificity in relation to the threshold changes was plotted for each of these antibiotics. This analysis was performed using Python programming language (Python Software Foundation. Python Language Reference, version 3.7., available at http:/ / www.python.org, accessed on 8 March 2021, scikit-learn library) and visualized using matplotlib [32].

Genomes of all isolates studied in this work have been deposited in GenBank and their accession numbers are shown in Supplementary Table S1.

The present study was approved by the ethics committee of the Principality of Asturias.

\section{Conclusions}

In summary, we found that OmpK36 alterations could play an important role on the resistance to carbapenems of OXA-48-producing K. pneumoniae, consistent with the fact that this porin constitutes the main pathway of entrance of these drugs into the cell. Although our study only includes isolates recovered from a single hospital, they represent a great variety of K. pneumoniae clones, and different mutations in ompK36 were found. As far as we know, our study represents the most extensive and complete molecular analysis of OXA-48-producing K. pneumoniae with alterations in the outer membrane porins. We have also demonstrated that these alterations do not affect the susceptibility to ceftazidime/avibactam, and thus this drug combination could be positioned as the choice therapy to combat the infections caused by this difficult to treat isolates. Finally, phenotypic detection of isolates carrying functional alterations on OmpK36 in clinical microbiology laboratories is important, and cefoxitin together with ertapenem/meropenem low inhibition zone diameters would strongly suggest alterations in this porin. 
Supplementary Materials: The following are available online at https: / www.mdpi.com/article/ 10.3390/antibiotics10101174/s1, Figure S1: Tridimensional analysis, assessed by the Phyre2 web, of OmpK36 protein structures of OXA-48-producing Klebsiella pneumoniae isolates displaying highlevel resistance to meropenem. This web was not able to create structures for Kp_HUCA_Bac_5, Kp_HUCA_Bac_24, Kp_HUCA_Bac_65, Kp_HUCA_Bac_85, Kp_HUCA_4 and Kp_HUCA_8 since the proteins predicted had less than 30 amino acids., Table S1: GenBank accession numbers of the genomes of OXA-48-producing Klebsiella pneumoniae isolates, Table S2: Microbiological features of OXA-48-producing Klebsiella pneumoniae isolates displaying meropenem MICs lower than $8 \mathrm{mg} / \mathrm{L}$.

Author Contributions: Conceptualization, J.F. and M.R.R.; methodology, J.F., P.L.-I., P.V. and T.S.-Z.; software, P.V.; resources, J.F.; writing-original draft preparation, P.L.-I. and J.F.; writing-review and editing, J.F. and M.R.R.; funding acquisition, J.F. All authors have read and agreed to the published version of the manuscript.

Funding: This research was funded by project FIS PI17-00728 (Fondo de Investigación Sanitaria, Instituto de Salud Carlos III, Ministerio de Economía y Competitividad, Spain), co-funded by the European Regional Development Fund of the European Union: A Way to Making Europe; and by Pfizer as an Independent Scientific Research Grant.

Institutional Review Board Statement: The study was conducted according to the guidelines of the Declaration of Helsinki, and approved by the Ethics Committee of the Principality of Asturias.

Informed Consent Statement: Not applicable.

Data Availability Statement: Data is contained within the article and/or supplemental materials. Genomes have been deposited in GenBank. Additional data are freely available on request from the corresponding author.

Conflicts of Interest: The authors declare no conflict of interest. The funders had no role in the design of the study; in the collection, analyses, or interpretation of data; in the writing of the manuscript, or in the decision to publish the results.

\section{References}

1. Vardakas, K.; Tansarli, G.; Rafailidis, P.; Falagas, M. Carbapenems versus alternative antibiotics for the treatment of bacteraemia due to Enterobacteriaceae producing extended-spectrum $\beta$-lactamases: A systematic review and meta-analysis. J. Antimicrob. Chemother. 2012, 67, 2793-2803. [CrossRef] [PubMed]

2. Tamma, P.; Han, J.; Rock, C.; Harris, A.; Lautenbach, E.; Hsu, A.; Avdic, E.; Cosgrove, S. Carbapenem therapy is associated with improved survival compared with piperacillin-tazobactam for patients with extended-spectrum $\beta$-lactamase bacteremia. Clin. Infect. Dis. 2015, 60, 1319-13125. [CrossRef] [PubMed]

3. Haidar, G.; Clancy, C.; Chen, L.; Samanta, P.; Shields, R.; Kreiswirth, B.; Nguyen, M. Identifying spectra of activity and therapeutic niches for ceftazidime-avibactam and imipenem-relebactam against carbapenem-resistant Enterobacteriaceae. Antimicrob. Agents Chemother. 2017, 61, e00642-17. [CrossRef]

4. Hamzaoui, Z.; Ocampo-Sosa, A.; Fernandez Martinez, M.; Landolsi, S.; Ferjani, S.; Maamar, E.; Saidani, M.; Slim, A.; MartinezMartinez, L.; Boutiba-Ben Boubaker, I. Role of association of OmpK35 and OmpK36 alteration and blaESBL and/or blaAmpC genes in conferring carbapenem resistance among non-carbapenemase producing Klebsiella pneumoniae. Int. J. Antimicrob. Agents 2018, 52, 898-905. [CrossRef]

5. Van Duin, D.; Doi, Y. The global epidemiology of carbapenemase-producing Enterobacteriaceae. Virulence 2016, 8, 460-469. [CrossRef]

6. Papp-Wallace, K.; Endimiani, A.; Taracila, M.; Bonomo, R. Carbapenems: Past, Present, and Future. Antimicrob. Agents Chemother. 2011, 55, 4943-4960. [CrossRef]

7. Rodríguez-Baño, J.; Gutiérrez-Gutiérrez, B.; Machuca, I.; Pascual, A. Treatment of infections caused by extended-spectrumbeta-lactamase-, AmpC-, and carbapenemase-producing Enterobacteriaceae. Clin. Microb. Rev. 2018, 31, e00079-17. [CrossRef] [PubMed]

8. Gutiérrez-Gutiérrez, B.; Salamanca, E.; de Cueto, M.; Hsueh, P.; Viale, P.; Paño-Pardo, J.; Venditti, M.; Tumbarello, M.; Daikos, G.; Cantón, R.; et al. Effect of appropriate combination therapy on mortality of patients with bloodstream infections due to carbapenemase-producing Enterobacteriaceae (INCREMENT): A retrospective cohort study. Lancet Infect. Dis. 2017, 17, 726-734. [CrossRef]

9. Poirel, L.; Potron, A.; Nordmann, P. OXA-48-like carbapenemases: The phantom menace. J. Antimicrob. Chemother. 2012, 67, 1597-1606. [CrossRef]

10. Pitout, J.; Peirano, G.; Kock, M.; Strydom, K.; Matsumura, Y. The Global Ascendency of OXA-48-Type Carbapenemases. Clin. Microb. Rev. 2019, 33, 00102-19. [CrossRef] [PubMed] 
11. Zhanel, G.; Lawson, C.; Adam, H.; Schweizer, F.; Zelenitsky, S.; Lagacé-Wiens, P.; Denisuik, A.; Rubinstein, E.; Gin, A.; Hoban, D.; et al. Ceftazidime-Avibactam: A Novel Cephalosporin/ $\beta$-lactamase Inhibitor Combination. Drugs 2013, 73, 159-177. [CrossRef]

12. García-Fernaández, A.; Miriagou, V.; Papagiannitsis, C.; Giordano, A.; Venditti, M.; Mancini, C.; Carattoli, A. An ertapenemresistant extended-spectrum- $\beta$-lactamase-producing Klebsiella pneumoniae clone carries a novel OmpK36 porin variant. Antimicrob. Agents Chemother. 2010, 54, 4178-4184. [CrossRef]

13. Netikul, T.; Kiratisin, P. Genetic characterization of carbapenem-resistant Enterobacteriaceae and the spread of carbapenem-resistant Klebsiella pneumonia ST340 at a university hospital in Thailand. PLoS ONE 2015, 10, e0139116. [CrossRef]

14. Tsai, Y.; Fung, C.; Lin, J.; Chen, J.; Chang, F.; Chen, T.; Siu, L. Klebsiella pneumoniae outer membrane porins OmpK35 and OmpK36 play roles in both antimicrobial resistance and virulence. Antimicrob. Agents Chemother. 2011, 55, 1485-1493. [CrossRef] [PubMed]

15. Clancy, C.; Chen, L.; Hong, J.; Cheng, S.; Hao, B.; Shields, R.; Farrell, A.; Doi, Y.; Zhao, Y.; Perlin, D.; et al. Mutations of the ompK36 porin gene and promoter impact responses of sequence type 258, KPC-2-producing Klebsiella pneumoniae strains to doripenem and doripenem-colistin. Antimicrob. Agents Chemother. 2013, 57, 5258-5265. [CrossRef] [PubMed]

16. Zhang, Y.; Jiang, X.; Wang, Y.; Li, G.; Tian, Y.; Liu, H.; Ai, F.; Ma, Y.; Wang, B.; Ruan, F.; et al. Contribution of $\beta$-lactamases and porin proteins OmpK35 and OmpK36 to carbapenem resistance in clinical isolates of KPC-2-producing Klebsiella pneumoniae. Antimicrob. Agents Chemother. 2013, 58, 1214-1217. [CrossRef] [PubMed]

17. Wong, J.; Romano, F.; Kelly, L.; Kwong, H.; Low, W.; Brett, S.; Clements, A.; Beis, K.; Frankel, G. OmpK36-mediated Carbapenem resistance attenuates ST258 Klebsiella pneumoniae in vivo. Nat. Com. 2019, 10, 3957. [CrossRef]

18. Lunha, K.; Chanawong, A.; Lulitanond, A.; Wilailuckana, C.; Charoensri, N.; Wonglakorn, L.; Saenjamla, P.; Chaimanee, P.; Angkititrakul, S.; Chetchotisakd, P. High-level carbapenem-resistant OXA-48-producing Klebsiella pneumoniae with a novel OmpK36 variant and low-level, carbapenem-resistant, non-porin-deficient, OXA-181-producing Escherichia coli from Thailand. Diag. Microb. Infect. Dis. 2016, 85, 221-226. [CrossRef]

19. Navon-Venezia, S.; Kondratyeva, K.; Carattoli, A. Klebsiella pneumoniae: A major worldwide source and shuttle for antibiotic resistance. FEMS Microb. Rev. 2017, 41, 252-275. [CrossRef]

20. Peirano, G.; Chen, L.; Kreiswirth, B.; Pitout, J. Emerging antimicrobial-resistant high-risk Klebsiella pneumoniae Clones ST307 and ST147. Antimicrob. Agents Chemother. 2020, 64, e01148-20. [CrossRef]

21. Cornaglia, G.; Mazzariol, A.; Fontana, R.; Satta, G. Diffusion of carbapenems through the outer membrane of Enterobacteriaceae and correlation of their activities with their periplasmic concentrations. Microb. Drug. Res. 1996, 2, 273-276. [CrossRef]

22. Sugawara, E.; Kojima, S.; Nikaido, H. Klebsiella pneumoniae major porins OmpK35 and OmpK36 allow more efficient diffusion of $\beta$-lactams than their Escherichia coli homologs OmpF and OmpC. J. Bacter. 2016, 198, 3200-3208. [CrossRef] [PubMed]

23. Nikaido, H. Molecular basis of bacterial outer membrane permeability revisited. Microb. Mol. Biol. Rev. 2003, 67, 593-656. [CrossRef] [PubMed]

24. Pagès, J.; Peslier, S.; Keating, T.; Lavigne, J.; Nichols, W.; Nichols, W. Role of the outer membrane and porins in susceptibility of $\beta$-lactamase-producing Enterobacteriaceae to ceftazidime-avibactam. Antimicrob. Agents Chemother. 2015, 60, 1349-1359. [CrossRef]

25. Martínez-Martínez, L.; Hernández-Allés, S.; Albertí, S.; Tomás, S.; Benedi, V.; Jacoby, G. In vivo selection of porin-deficient mutants of Klebsiella pneumoniae with increased resistance to cefoxitin and expanded-spectrum-cephalosporins. Antimicrob. Agents Chemother. 1996, 40, 342-348. [CrossRef]

26. European Committee on Antimicrobial Susceptibility Testing (EUCAST). Breakpoint Tables for Interpretation of Mics and Zone Diameters. Version 11.0. 2021. Available online: https://www.eucast.org/clinical_breakpoints/ (accessed on 2 March 2021).

27. Rodríguez-Lucas, C.; Rodicio, M.; Rosete, Y.; Fernández, J. Prospective evaluation of an easy and reliable work flow for the screening of OXA-48-producing Klebsiella pneumoniae in endemic settings. J. Hosp. Infect. 2020, 105, 659-662. [CrossRef]

28. Zerbino, D.; Birney, E. Velvet: Algorithms for de novo short read assembly using de Bruijn graphs. Genome Res. 2008, 18, 821-829. [CrossRef]

29. CGE. Center for Genomic Epidemiology. 2020. Available online: http://www.genomicepidemiology.org (accessed on 25 January 2021).

30. Madeira, F.; Park, Y.; Lee, J.; Buso, N.; Gur, T.; Madhusoodanan, N.; Basutkar, P.; Tivey, A.; Potter, S.; Finn, R.; et al. The EMBL-EBI search and sequence analysis tools APIs in 2019. Nucl. Acids Res 2019, 47, W636-W641. [CrossRef]

31. Waterhouse, A.; Procter, J.; Martin, D.; Clamp, M.; Barton, G. Jalview Version 2-A multiple sequence alignment editor and analysis workbench. Bioinformatics 2009, 25, 1189-1191. [CrossRef]

32. Hunter, J. Matplotlib: A 2D graphics environment. Comput. Sci. Eng. 2017, 9, 90-95. [CrossRef] 